Journal for

ImmunoTherapy of Cancer

\title{
Severity of COVID-19 in patients with lung cancer: evidence and challenges
}

\author{
Antonio Passaro (D) , ${ }^{1}$ Christine Bestvina ${ }^{2}$ Maria Velez Velez, ${ }^{3}$ \\ Marina Chiara Garassino, ${ }^{4}$ Edward Garon, ${ }^{5}$ Solange Peters ${ }^{6}$
}

To cite: Passaro A, Bestvina C, Velez Velez M, et al. Severity of COVID-19 in patients with lung cancer: evidence and challenges. Journal for ImmunoTherapy of Cancer 2021;9:e002266. doi:10.1136/ jitc-2020-002266

$\mathrm{AP}, \mathrm{CB}$ and MVV contributed equally.

MCG, EG and SP contributed equally.

Accepted 26 January 2021

Check for updates

(C) Author(s) (or their employer(s)) 2021. Re-use permitted under CC BY-NC. No commercial re-use. See rights and permissions. Published by BMJ.

${ }^{1}$ Division of Thoracic Oncology, European Institute of Oncology IRCCS, Milan, Italy

${ }^{2}$ Department of Hematology/ Oncology, University of Chicago Medicine, Chicago, Illinois, USA ${ }^{3}$ Division of Hematology/ Oncology, David Geffen School of Medicine, University of California, Los Angeles, California, USA

${ }^{4}$ Department of Medical Oncology, Fondazione IRCCS Istituto Nazionale dei Tumori,

Milan, Italy

${ }^{5}$ David Geffen School of Medicine, UCLA, University of California Los Angeles, Los Angeles, California, USA

${ }^{6}$ Department of Oncology, Centre Hospitalier Universitaire Vaudois, CHUV, Lausanne, Switzerland

Correspondence to

Solange Peters;

solange.peters@chuv.ch

\section{ABSTRACT}

Cancer patients are highly vulnerable to SARS-CoV-2 infections due to frequent contacts with the healthcare system, immunocompromised state from cancer or its therapies, supportive medications such as steroids and most importantly their advanced age and comorbidities. Patients with lung cancer have consistently been reported to suffer from an increased risk of death compared with other cancers. This is possibly due to the combination of specific pathophysiological aspects, including underlying pulmonary compromise due to smoking history and the increased specific pressures on respiratory healthcare services caused by the related pandemic. Rationally and safely treating patients with lung cancer during the pandemic has become a continuous challenge over the last year. Deciding whether to offer, modify, postpone or even cancel treatments for this particular patient's population has become the crucial recurrent dilemma for lung cancer professionals. Chemotherapy, immunotherapy and targeted agents represent distinct risks factors in the context of COVID-19 that should be balanced with the short-term and long-term consequences of delaying cancer care. Despite the rapid and persistent trend of the pandemic, declared by WHO on March 11, 2020, and still ongoing at the time of writing (January 2021), various efforts were made by oncologists worldwide to understand the impact of COVID-19 on patients with cancer. Adapted recommendations of our evidencebased practice guidelines have been developed for all stakeholders. Different small and large-scale registries, such as the COVID-19 and Cancer Consortium (CCC19) and Thoracic Cancers International COVID-19 Collaboration quickly collected data, supporting cancer care decisions under the challenging circumstance created by the COVID-19 pandemic. Several recommendations were developed as guidance for prioritizing the various aspects of lung cancer care in order to mitigate the adverse effects of the COVID-19 healthcare crisis, potentially reducing the morbidity and mortality of our patients from COVID-19 and from cancer. These recommendations helped inform decisions about treatment of established disease, continuation of clinical research and lung cancer screening. In this review, we summarize available evidence regarding the direct and indirect impact of the COVID-19 pandemic on lung cancer care and patients.

\section{INTRODUCTION}

Patients with cancer have been disproportionately affected by the COVID-19 pandemic. Although data collected are heterogeneous due to patients' demographics, variable testing availability or policy, and cancer patients' management guidelines over time, $1 \%-8 \%$ of patients hospitalized with COVID-19 had an history of hematological malignancies or solid tumors. ${ }^{1}$ Available data confirm that patients with cancer are possibly more likely to be infected by SARS-CoV-2 and, more importantly, definitively prone to develop severe complications defined here as admission in an intensive care unit (ICU), the need for mechanical ventilation (MV) or death as a result of COVID-19. The mortality rate in patients with cancer has been reported to be $25 \%-30 \% .^{1-3}$

Large datasets demonstrate that cancer is independently associated with mortality in hospitalized patients with COVID-19. ${ }^{4}$ Not all cancers appear to have equal risks of morbidity and mortality, with a mortality range from $8 \%$ to $30 \% .^{5}$

\section{THE SPECIFIC CASE OF LUNG CANCER}

Patients with lung cancer and those with hematological malignancies appear to be at the highest risk of death from to SARS-CoV-2 infection. ${ }^{6}$ In contrast to hematologic malignancies, profoundly immunosuppressive therapy is not typical in lung cancer.

Lung cancer represents a clinical scenario characterized by an increased risk of pulmonary complications, severe lung injury and high mortality from COVID-19, due to pathophysiological, clinical and treatment-related risk factors. ${ }^{7}$

A significant interplay of factors frequently associated with lung cancer, including smoking-related lung damage, significant cardiovascular and respiratory comorbidities, and older age, induce higher severity of SARS-CoV-2 infection. ${ }^{7-9}$ Patients with lung cancer share fragility related to defective pulmonary and alveolar architecture due to previous thoracic surgery or radiotherapy and/or malignant airway obstruction that may also predispose to more severe infections. 
The alterations of alveolar epithelium and pulmonary vessels lead to tumor microenvironment modifications, including an increase in immune cell and tissue-resident macrophages infiltration, critical for innate immunity and inflammation. ${ }^{110}$

This widespread immune component within alveolar epithelium poses a higher risk for cytokine release. In addition, the alveolar damage and fibrin deposits represent risk factors for thrombosis of small and large pulmonary vessels. ${ }^{11}$ These two pathogenetic pillars have been postulated to be the major steps in leading to the development of severe lung injury and acute respiratory distress syndrome (ARDS) during the SARS-CoV-2 infection. ${ }^{11}$

Smokers are five times more likely to contract influenza than non-smokers, and in particular, smoking history has been related to a higher incidence and severity of SARS-CoV-2 infection. ${ }^{11}$ Structural and immunologicinduced modifications are the two main sources of tobacco-related damage that accounts for susceptibility to infections. Changes in peribronchiolar homeostasis and inflammation additionally impair the building of an efficient antiviral or bacterial immune response required for infection clearance. ${ }^{12-14}$ Smokers showed high risk $(\times 2.4$ times) of COVID-19 severe events (ICU admission, MV or death) compared with non-smokers $(\mathrm{RR}=2.4,95 \% \mathrm{CI}$ 1.43 to 4.04$){ }^{14}$

Additionally, a cumulative risk for severe COVID-19 injury is related to long-term tobacco-related lung damage, including chronic obstructive pulmonary disease (COPD) and lung cancer. ${ }^{7}$

Finally, the all-cause case-fatality rate in patients with cancer after SARS-CoV-2 infection was significantly associated with increasing age, rising steadily from 65 years, encompassing the majority of patients with lung cancer. ${ }^{15}$

The interaction between these pathological and clinical factors, in addition to the complex role of antineoplastic therapy and concomitant medications, define patients with lung cancer as an extremely vulnerable patient subgroup at the time of COVID-19 pandemic, for severe illness.

Reflecting on the unmet needs characterizing the management of non-communicable diseases globally, patients with cancer, and in particular lung cancer, are additionally suffering from the disruption of healthcare service due to COVID-19, which is significantly amplifying the specific negative prognostic impact of this novel disease on our patients. ${ }^{16}$

As described across registries, the well-known dismal prognosis of lung cancer combined with reduced cancer services and simultaneously reduced ICU capacity lead to ICU triage strategies that often disadvantage patients with lung cancer and significantly augment their mortality. ${ }^{3517}$

\section{MORTALITY RATE ACROSS MAIN REGISTRIES AND SERIES}

During the COVID-19 pandemic, several real-world datasets investigated the risk for hospitalization, complications and death in patients with cancer. Data were generally retrospectively collected through multicenter registry-based observational cohort studies worldwide ${ }^{618}$ (table 1).

Although the fast enrollment and the heterogeneity of patient populations are prone to potential biases, the results consistently confirmed that patients with cancer represent a significantly vulnerable group to SARS-CoV-2 infection. ${ }^{18}$

The impact of COVID-19 on patients with thoracic malignancies-and in particular lung cancer-was evaluated through two different approaches: non-diseaseoriented studies including patients with or without a diagnosis of solid or hematologic tumors; and diseasespecific studies, planned and developed to investigate the impact of SARS-CoV-2 infection on patients with thoracic cancer.

\section{Registry data from China}

In the largest report from China, a multicenter retrospective cohort study including 13077 patients with COVID19, 23 had a history of lung cancer, although only five were metastatic. In this subgroup, $78 \%$ of patients showed a severe clinical outcome with a mortality rate of $39 \%$. Overall, this series showed that patients with cancer were more likely to have severe illness. It also confirmed that ECOG Performance Status (PS) $\geq 2$, older age and advanced or metastatic stage were all associated with an increase in mortality rate. ${ }^{19}$

Dai et al reported a multicenter retrospective analysis including 105 hospitalized patients with cancer from 14 hospitals in Hubei Province, China, the epicenter of the first COVID-19 outbreak. ${ }^{20}$ Lung cancer accounted for $20.9 \%(22 / 105)$ of these patients, with a death rate of $18.1 \%$ (4/22), an ICU admission rate of $27.2 \%(6 / 22)$, risks of severe/critical symptoms in $50 \%(11 / 22)$, and utilization of invasive mechanical ventilation (IMV) of $18.1 \%$ (4/22). In patients affected by lung cancer, the average time (means/SD days) from initial onset of COVID-19 symptoms to death (16.75/8.17), ICU admission $(10.00 / 6.82)$, critical symptoms $(8.55 / 5.71)$ and IMV (14.50/6.98) were at least a week from symptom onset. Data from this first large cohort study raised two very interesting issues: compared with all other patients, patients with primary or metastatic tumors in the lungs were more prone to rapid clinical deterioration on SARS-CoV-2 infection and patients who underwent cancer surgery showed higher complication and mortality rates. ${ }^{20}$

\section{Retrospective analysis from the Veneto region of Italy}

In an extensive retrospective epidemiological study, developed during the first peak of COVID-19 pandemic in the Veneto region (Northeast of Italy), investigators collected data from 84,246 consecutive Italians tested for SARS-CoV-2 to evaluate the prevalence of cancer and clinical outcomes of viral infection. ${ }^{21}$ Among the 9275 SARS-CoV-2 positive patients, 723 had a cancer diagnosis $(7.8 \%)$, which was associated with a high rate of 


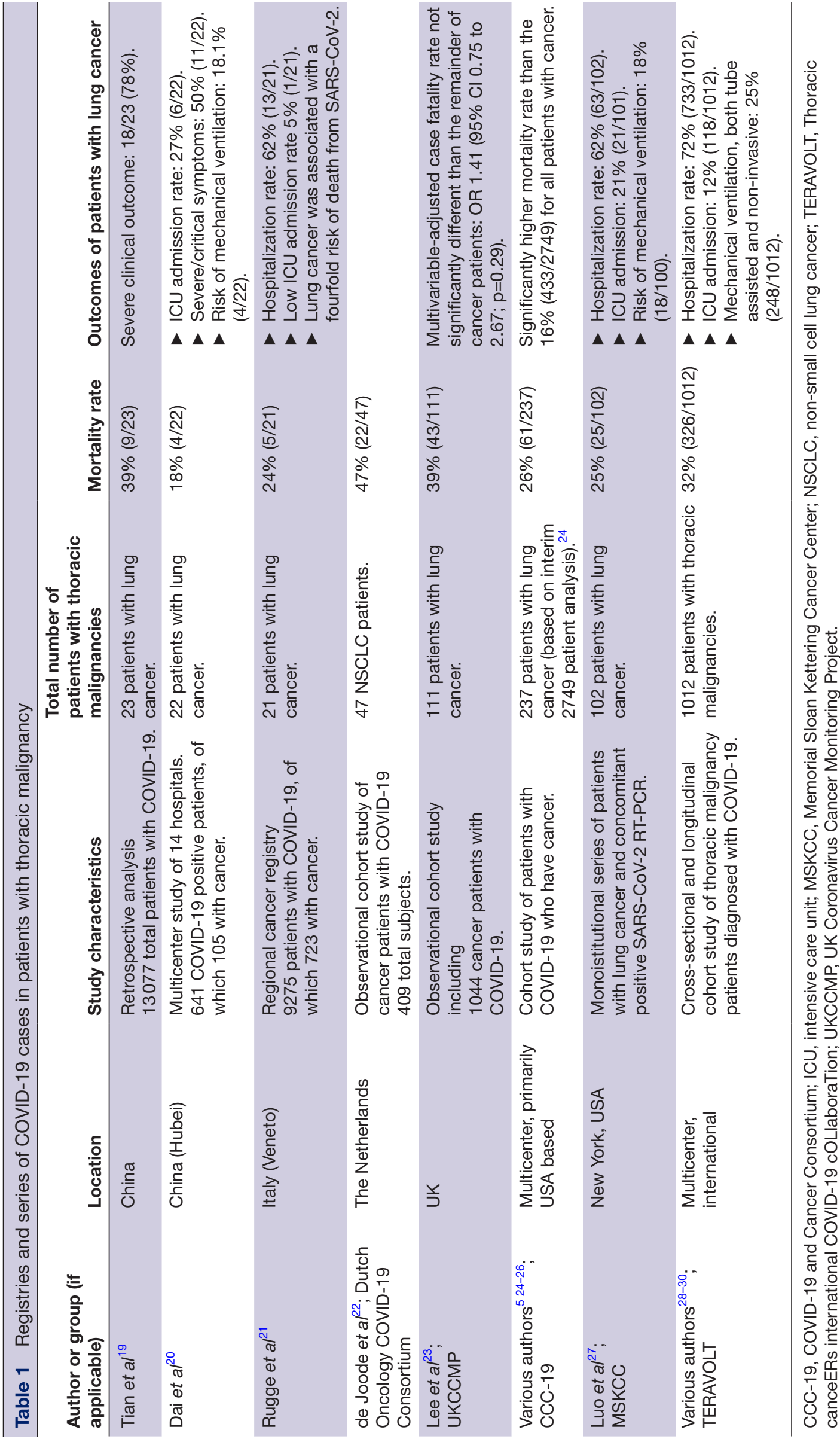


hospitalization (56.6 vs $34.4 \%$ ) and mortality ( $14.7 \%$ vs $4.5 \%$ ) particularly for males and elderly patients. Overall, 21 patients had a lung cancer diagnosis, with a mortality rate of $23.8 \%(5 / 21)$ and a hospitalization rate of $61.9 \%$ $(13 / 21)$. Of note, only one patient with lung cancer was admitted to ICU, while in the overall study population, ICU admission rate for COVID-19 was not associated with cancer. Lung cancer was associated with a fourfold risk of death due to SARS-CoV-2 infection, although the patient population was too small to draw robust conclusions. ${ }^{21}$

\section{Dutch Oncology COVID-19 Consortium (DOCC)}

The Dutch Oncology COVID-19 consortium developed the DOCC registry. Overall, 409 patients with a cancer diagnosis or cancer treatment in the past 5 years were included in this analysis. In this registry, $13.4 \% \quad(n=47)$ patients reported a diagnosis of lung cancer, showing a mortality rate (fatal event) of $47 \%(22 / 47) .{ }^{22}$ In a multivariate analysis, de Joode and colleagues ${ }^{22}$ showed that lung cancer $(p=0.003)$, male gender $(p=0.035)$ and age $\geq 65$ years $(p<0.001)$ remained independent risk factors for a fatal outcome of COVID-19.

\section{UK Coronavirus Cancer Monitoring Project (UKCCMP)}

In contrast to previously reported findings, in a nondisease-oriented registry, the UKCCMP database did not observe an increased case-fatality rate for patients with lung cancer $(0.39,43 / 111$ patients with lung cancer) compared with other cancers, despite 43 deaths. The authors proposed that lung cancer might not be considered as a specifically susceptible group, keeping in mind that these results may have been affected by the relatively small sample size. Lee $e t a t^{23}$ confirmed that the all-cause case-fatality rate in patients with cancer after SARS-CoV-2 infection was significantly associated with increasing age, from 0.10 in patients aged $40-49$ to 0.48 in those aged 80 years and older.

\section{COVID-19 and Cancer Consortium (CCC-19)}

In addition to the Chinese and European registries, data about adults with a current or past diagnosis of cancer and COVID-19 were collected in the well-structured US-based CCC-19 cohort study. ${ }^{5}$ The preliminary comprehensive results of the CCC-19 was based on 928 patients, including $91(10 \%)$ with lung cancer, demonstrated an overall mortality rate of $13 \%$, a hospitalization rate of $50 \%$ including $14 \%$ of ICU admission, and $12 \%$ rate of mechanical ventilation. ${ }^{5}$ The updated analysis based on 2749 evaluable patients showed a high mortality rate of $26 \%$ for patients with lung cancer $(61 / 237)$, significantly increased compared with breast cancer $(8 \%)$, prostate cancer $(18 \%)$, colorectal cancer $(19 \%)$ or lymphoma (22\%), with an updated $16 \%(433 / 2749)$ mortality for the whole CCC-19 cohort. $^{24}$

The CCC-19 data were updated step by step during the pandemic, from 928 to 3899 evaluable patients. Analysis confirmed that different independent factors are associated with 30-day mortality:
- General factors (eg, older age, male sex and number of major comorbidities (two vs none)).

- Cancer-specific factors (eg, ECOG PS $>1$, active or progressive cancer).

- Laboratory variables (eg, low absolute lymphocyte count, high or low absolute neutrophil count, low platelet count, abnormal creatinine, D-dimers, troponin, and C reactive protein). ${ }^{24-26}$

As discussed previously, completing some non-diseasespecific evaluation, several specific thoracic canceroriented registries or cohort study evaluated the impact of COVID-19 in this specific cancer population.

\section{MSKCC retrospective analysis}

In a USA single-center retrospective series, Luo and colleagues $^{27}$ investigated the thresholds of COVID-19 severity in lung cancer. Among the 102 evaluable patients, most with metastatic or active lung cancer (72\%), the rate of hospitalization was $62 \%$, and the overall mortality rate was $25 \%$, even though deaths attributed to COVID-19 constituted only $11 \%$ of all deaths. In addition, for patients that required ICU admission (21\%), $72 \%$ died. Pulmonary comorbidities (eg, COPD) or smoking history were the greatest determinants of COVID-19 severity. Cancer-specific features, including prior thoracic radiation or surgery or the presence of active/metastatic disease, did not appear to impact the severity of COVID-19.

In another report by Luo et $a l^{17}$ the authors evaluated the impact of the immunotherapy in patients with lung cancer and concomitant COVID-19 diagnosis, to investigate whether programmed death 1 (PD-1) blockade affects severity of illness from patients with COVID-19 in patients with lung cancer. The results, based on 69 consecutive patients with lung cancer, showed that PD-1 exposure was not associated with increased severity of COVID-19.

\section{Thoracic canceERs international COVID-19 19 cOLlaboraTion (TERAVOLT)}

A structured analysis of patients with lung cancer and COVID-19 was investigated in the TERAVOLT registry. The study enrolled patients with thoracic cancer (nonsmall cell lung cancer (NSCLC), mesothelioma, thymic epithelial tumors and other pulmonary neuroendocrine neoplasms) with either confirmed COVID-19 infection or clinically diagnosed infection. ${ }^{28}$

The updated results based on 1012 patients showed higher mortality (32\%) and hospitalization rate $(72 \%)$, compared with a dramatic low ICU admission (12\%) and mechanical ventilation rate $(7 \%) .{ }^{29}$

As reported in the CCC-19 results, TERAVOLT confirmed the COVID-19 related general risk factors for mortality, including age and comorbidities as well as cancer-specific factors, namely PS ( $\geq 2 \mathrm{vs}<2)$, and the use of steroids prior to COVID-19 diagnosis. ${ }^{28-30}$ 


\section{CONCLUSIONS FROM REGISTRY DATA}

Although data discussed are heterogeneous by nature due to different study designs and variable medical practices at the time of COVID-19, all results consistently confirm that lung cancer predicts a worse outcome on SARS-CoV-2 infection, as described by a higher rate of hospitalization and ARDS and a significantly higher mortality rate. ${ }^{31}$

\section{LUNG CANCER TREATMENT AND COVID-19}

The primary goals of the treatment of patients with lung cancer during the COVID-19 pandemic are to minimize the risk of patient exposure as well as the risks of complication should they contract COVID-19, while managing lung cancer, which has a high risk of mortality. ${ }^{32}$ Early in the pandemic, when there were shortages of personal protective equipment, limited COVID-19 testing supplies, and many regions of the world saw overwhelmed healthcare systems, lung cancer surgeries were delayed and systemic treatments were canceled. Fortunately, additional data have been gathered from TERAVOLT, CCC-19, and others, allowing providers and patients to make a risk-benefit analysis of proceeding with treatment versus delaying or omitting.

In this manuscript, we analyzed data from the main registries and series about the impact of specific cancer treatment for lung cancer during the pandemic. During these troubled times, different evidence-based recommendations (eg, American Society of Clinical Oncology (ASCO), ${ }^{33}$ European Society for Medical Oncology $(\text { ESMO })^{34} 35$ and International Association for the Study of Lung Cancer (IASLC) ${ }^{32}$ were developed to improve the management of patients with lung cancer and to mitigate the pandemic impact on our patients.

\section{Surgery}

While surgical resection strategies for lung cancer remain the same, many logistical challenges arise in weighing the risks and benefits to delaying resection based on the severity of the COVID-19 pandemic. Limited resources including hospital beds have led to delays of non-urgent surgeries, particularly non-oncological elective interventions. Perioperative COVID-19 has been associated with mortality rates as high as $10 \%-24 \%$ in the overall surgery population. ${ }^{36}{ }^{37}$ For patients undergoing surgery for lung cancer, the UK Lung Cancer Coalition's Clinical Advisory Group stated that increased mortality as high as 40\%-50\% has been seen in patients who contracted COVID-19 following surgery for lung cancer. ${ }^{38}{ }^{39}$ To reduce the risk of operation during the incubation period for COVID19, patients should receive COVID-19 testing prior to surgery. ${ }^{32}$

The ESMO recommends keeping all NSCLC surgeries as a priority and that surgical delays should not last more than 6-8 weeks. ${ }^{35}$ The ASCO, however, recommends clinicians and patients make individual determinations of risk and benefit in proceeding with surgery. ${ }^{40}$ The American College of Surgeons has published guidelines on triaging elective cases for surgical care during the COVID-19 pandemic and includes characteristics such as solid versus non-solid component, size of the presumed lung cancer, node positivity, and completion of induction chemotherapy. ${ }^{41}$ All of this information can help triage patients treatment, as well as help thoracic surgeons advocate for their patients in the context of COVID-19.

\section{Radiation therapy}

The European Society for Radiotherapy and Oncology and the American Society for Radiation Oncology partnered to author: 'Practice recommendations for lung cancer radiotherapy during the COVID-19 pandemic'. ${ }^{4}$ Treatment recommendations are given for a variety of clinical scenarios, from stage I-III NSCLC, prophylactic cranial irradiation for small cell lung cancer (SCLC), and palliative radiation for NSCLC. Recommendations are given for both an 'early pandemic scenario', where risk mitigation of both the patient and radiotherapy staff are balanced with the treatment of lung cancer, as well as a 'later pandemic scenario', where resources are limited requiring patient triage. Hypofractionated radiation schedules have been recommended to reduce the number of visits to heathcare facilities, as well as possible patient and staff exposure to COVID-19. ${ }^{43}$ Additional recommendations have included avoiding twice daily radiation, the use of single fraction radiation in the palliative setting, and the delay or omission of prophylactic cranial irradiation for patients with SCLC. ${ }^{44}$

\section{Chemotherapy}

In the original TERAVOLT analysis of 200 patients, treatment with chemotherapy alone was associated with increased risk of death (HR 2.54, 95\% CI 1.09 to 6.11 ) ${ }^{28} \mathrm{In}$ an updated analysis of 1012 patients presented at ESMO, patients receiving chemotherapy alone or not receiving treatment had worse outcomes compared with patients receiving immunotherapy, chemoimmunotherapy, or targeted therapy (HR 1.4, 95\% CI 1.02 to $2.0, \mathrm{p}=0.03){ }^{29}$ In a single center analysis of 102 patients with lung cancer treated at Memorial Sloan Kettering, no effect of recent systemic therapy was seen on COVID-19 outcomes, including chemotherapy or chemoimmunotherapy. ${ }^{27}$ In the CCC-19 registry, which includes patients of multiple tumor types, the highest 30-day mortality by treatment type was chemoimmunotherapy at $30 \%$ 30-day mortality. ${ }^{5}$ Patients receiving chemotherapy or chemoradiation had a 30 -day mortality of $18 \%$ (Wise-Draper ESMO) ${ }^{45}$ However, it is difficult to know how to interpret these data given the inclusion of multiple tumor types and the wide variety of systemic therapies included in the analysis. In fact, 182 distinct drugs were included in the analysis. Additionally, the diseases and patients' characteristics, including comorbidities, defining each treatment opportunity in lung cancer-like for example chemotherapy without immunotherapy-will per se define a precise disease scenario that can only incompletely be accounted and adjusted for. 
In addition to the risk of COVID-19 infection for patients with cancer, delays in cancer care can also negatively affect patients' cancer course. The average delay of oncologic treatment for patients in the TERAVOLT registry was 21 days. ${ }^{29}$ The Centers for Disease Control and Prevention (CDC) recommends immunocompetent individuals with COVID-19 quarantine for 10 days, while those considered immunocompromised quarantine for 20 days. ${ }^{46}$ It remains to be seen how treatment delays will affect patient's cancer outcomes. ${ }^{47}$

\section{Immunotherapy}

Early in the pandemic, there was concern that immune checkpoint inhibitors (ICIs) may worsen the complications of COVID-19. The concern was that ICIs may increase the cytokine storm causing ARDS of COVID-19, as well as the possibility of overlapping pneumonitis from ICIs and COVID-19. ${ }^{48}$ However, the results of multiple registries including TERAVOLT, ${ }^{28-30}$ the CCC19, ${ }^{54}$ as well as 102 patients with lung cancer treated at Memorial Sloan Kettering Cancer Center, ${ }^{17} 27$ all demonstrated that patients treated with ICIs alone, without chemotherapy, had outcomes equivalent or better to those receiving other cancer treatments. Given this information, for patients who have high PDL1 expression $\geq 50 \%$, treatment with immunotherapy alone should be strongly considered over chemoimmunotherapy. ${ }^{32}$

Alternative dosing of immunotherapy regimens, which are administered less frequently, can and should be considered. This can limit both patient and provider exposures, while retaining efficacy, thereby optimizing the risk-benefit ratio. ${ }^{32} 35$ Pembrolizumab $400 \mathrm{mg}$ given every 6 weeks has been approved by both the European Medicines Agency (EMA) as well as the US Food and Drug Administration (FDA) ${ }^{49}$ Nivolumab can be dosed $480 \mathrm{mg}$ every 4 weeks as opposed to every other week. ${ }^{50}$ Atezolizumab is able to be dosed every 4 weeks at $1680 \mathrm{mg}^{51}$ Durvalumab dosing of $1500 \mathrm{mg}$ every 4 weeks was initially approved and has demonstrated efficacy in the small cell population. ${ }^{52}$ The every 4 -week dosing can be considered for the stage III NSCLC consolidation setting as well. ${ }^{32}$

Pneumonitis present on CT scans for patients with lung cancer has traditionally had a differential of immunotherapy pneumonitis, radiation pneumonitis, or progressive lung cancer depending on the clinical context. COVID-19 pneumonitis has also been reported on imaging and can be difficult to differentiate based on imaging alone. CT findings suggestive of COVID-19 should lead to an appropriate infectious workup for COVID-19 while taking into account the remaining differential. ${ }^{32} 53$

\section{Targeted therapy}

The treatment of NSCLC with tyrosine kinase inhibitors (TKIs) has become much more common over the last decade and has led to significant improvements in the 5 -year overall survival of patients with lung cancer. In the initial TERAVOLT analysis of 200 patients with thoracic malignancies, 28 patients were on TKI alone. These patients were less likely to be hospitalized (OR 0.24, $95 \%$ CI 0.077 to 0.708$).{ }^{28}$ In expanded analysis presented at both ASCO and ESMO, patients receiving targeted therapies do not have increased risk of death in multivariate analysis. ${ }^{29} 30$ The degree to which this relatively favorable outcome is related to the mechanisms of these therapies as opposed to the different demographics of the patients who use them (younger, non-smokers) is not fully understood.

Similar to immunotherapy, TKIs are known to cause interstitial-like pneumonitis at varying rates and can be difficult to distinguish from COVID-19 induced pneumonia. The clinical picture of new or progressive cough, dyspnea, and/or fever can also be similar. Patients should receive COVID-19 based testing if there is either radiographic or clinical concern. ${ }^{73253}$

\section{IMPACT OF COVID-19 PANDEMIC ON LUNG CANCER RESEARCH}

The COVID-19 pandemic has greatly affected the care of patients with lung cancer and has affected multiple aspects of cancer research. ${ }^{54}$ Since the start of the pandemic, there has been a decrease in clinical trial enrollment, and several active trials have been placed on hold. ${ }^{55}$ While data demonstrating the effect of COVID-19 specifically on lung cancer research is scant, several studies show that cancer research in general has been compromised. ${ }^{56-63}$ Cancer clinical trials have been at the focus of these studies, but it is essential to highlight that translational and observational studies have also been affected. ${ }^{57}$ The mechanisms by which research has been compromised include decreased funding, operational concerns with tissue collection and biobanking, holds on clinical trials, reduced workforce, and reduced networking opportunities for international collaborations. ${ }^{161}$

In an effort to help sustain cancer research during the pandemic, the National Institute of Health in the USA has implemented changes in its policies to increase flexibility around research funding, such as including administrative supplements to existing grant awards, extending deadlines for grant applications, allowing salary support through grants, and extending eligibility periods for early-stage trainees. However, other significant funding sources, such as philanthropic donations, have dramatically decreased. ${ }^{1}$ As such, organizations that depend on these donations to allocate grant funds, like the American Cancer Society, the Canadian Cancer Society, and Cancer Research UK, have had to significantly reduce their budget for research funding. ${ }^{60}{ }^{62}$ Despite these budget cuts, there have been ongoing efforts to continue cancer research and determine the impact of COVID-19 on patients with lung cancer (NCT04538456).

Operational challenges regarding obtaining, keeping, and processing specimens for translational and correlative studies have been profound. With the surge of COVID-19, patients requiring hospitalization have increased, leading to decreased hospital capacity and 
a subsequent postponement of surgeries and procedures. ${ }^{59} 64$ This has compromised cancer care and has affected the acquisition of specimens for translational and correlative studies. ${ }^{58} 64$ Since patients with lung cancer are at a higher risk of infection with SARS-CoV-2 than the general population, there are concerns with the handling of specimens from patients that test positive for the virus. ${ }^{64}$ These specimens are potentially infectious and require special procedures. ${ }^{58} \mathrm{~A}$ study evaluating the effect of COVID-19 on cancer biobanks showed that NCTN biobanks saw a dramatic decrease in both biospecimen receipt and distribution of $40 \%$ and $60 \%$, respectively. Finally, multiple cancer research laboratories have had to shut down because of local mandates, stay at home orders and to comply with social distancing strategies. This has lead to the loss of experimental resources such as patientderived xenografts, transgenic mice and cell lines. Other resources have been repurposed for COVID-19 research, like the evaluation of anticancer agents for the treatment of COVID-19. ${ }^{576566}$ These shutdowns will lead to delays in cancer research and potentially important discoveries. ${ }^{5767}$

While the effect of the COVID-19 on lung cancer trials specifically has not been reported, cancer trials in general have been severely disrupted since the start of the pandemic. ${ }^{1}$ For instance, cancer centers have generally prioritized certain clinical trials based on their evidence of clinical activity. ${ }^{66}$ As such, phase I trials have been disproportionately held. ${ }^{65}$ Suspension of clinical activities has affected the ability to recruit patients, comply with protocol-mandated visits, and has affected the acquisition of laboratory and radiological data that are essential for both trial endpoints and correlative studies. ${ }^{54}$ Many institutions have halted research-only blood draws and tissue collection and are only collecting samples that directly affect primary trial endpoints as long as patient safety is not compromised in doing so. ${ }^{68} 69$ An analysis of data obtained from a combination of surveys, IQVIA, clinicaltrials.gov, and interviews revealed that only around $15 \%-20 \%$ of trials conducted in Europe and the USA were enrolling patients at the usual rate. ${ }^{62}$ Additionally, $20 \%$ of clinical trials in Asia and the US were not enrolling patients when this survey was conducted. For investigators who continued to conduct trials, the greatest barriers to enrollment included the need for in-person patient evaluation and intravenous administration of the investigational agent. Most investigators reported reaching out to their ethic committees regarding concerns around staff safety $(30 \%)$ and protocol deviations (27\%). Regarding new clinical trials, investigators' main concerns included patient follow-up and type (eg, target agents, chemotherapy, immunotherapy or combination of these with some investigational agents) and route of administration of the agent (oral vs intravenous or subcutaneous). Sixty per cent of interviewees rated the pandemic as having a 'moderate' or 'high' impact on their trials, and $80 \%$ of respondents anticipated protocol deviations and concerns regarding missing data. This study also reported that around 200 clinical trials were suspended during
April and May. ${ }^{62}$ Similarly, a survey of 64 investigators conducted by ASCO evaluated the pandemic's impact at a clinical trial level in both academic and community centers. Fifty per cent of respondents reported discontinuation of research-only laboratory collections, $60 \%$ reported halting screening and enrollment, and most reported that initiation visits had to be conducted remotely. ${ }^{70}$

The pandemic has changed the way clinical research is being conducted. Investigators have had to develop innovative ways to continue to conduct cancer research. For instance, investigators are now using telemedicine for patient follow-up as well as electronic methods for obtaining consent remotely. ${ }^{62}$ Other strategies to mitigate direct patient contact have included the shipment of investigational agents to the patients' homes with administration supervision via telehealth. ${ }^{54}$ Furthermore, both the US FDA and the EMA released guidelines to help investigators, sponsors, and institutional review boards (IRBs) navigate clinical trials during this health crisis. These guidelines address issues such as the collection and reporting of data, different modalities for patient follow-up, and risk-benefit evaluations of continuing versus halting trials. ${ }^{71} 72$ The US FDA requested that sponsors increase flexibility regarding protocols and authorized remote communication with patients for follow-up that should be reflected in protocol amendments. ${ }^{66}$

To conclude, the pandemic has affected several aspects of cancer research, from preclinical studies to clinical trials. These are likely to decrease cancer discovery both in the short and long term. ${ }^{57}$ However, investigators have circumvented some of these difficult circumstances using different strategies that could change the way cancer research is conducted moving forward. ${ }^{54}$

\section{CONCLUSION}

The SARS-CoV-2 pandemic has severely affected patients with lung cancer and impaired the progress of lung cancer research. Patients with lung cancer are at an increased risk of becoming infected with the virus and experience higher morbidity and mortality than the general population. ${ }^{3}$ At the beginning of the pandemic, patients with lung cancer were deprioritized for the ICU admission or mechanical ventilation, as confirmed by the TERAVOLT data, with an increasing rate of death. To date, based on the acquired knowledge on the impact of COVID-19 in patients with cancer, this approach appears out of order, confirming that patients with lung cancer should not be excluded from ICU beds and should be prioritized to receive a prompt and comprehensive care, as other patients.

Despite challenges posed by the pandemic, there has been an unprecedented international effort to understand the impact of COVID-19 on this patient population. Cancer societies, including ESMO and ASCO, have released statements and guidelines to help clinicians manage patients with lung cancer during this health 
crisis. ${ }^{35} 40$ Studies such as the TERAVOLT analysis and CCC19 have provided essential information on the effect of different treatment modalities on patients with lung cancer in the context of COVID-19. ${ }^{58}$ These analyses provide detailed guidance on the risk of different treatment strategies, with a goal to improve outcomes in patients with lung cancer infected with the virus. Regarding the continuation of clinical research, the FDA and EMA have also released guidelines for continuing clinical research during the pandemic. They have urged sponsors to increase their flexibility and allow telemedicine and remote monitoring for patient follow-up of patients enrolled in clinical trials. ${ }^{71} 72$

As the number of COVID-19 cases continues to surge across the globe, it is becoming increasingly evident that the incidence of COVID-19 in patients with lung cancer will continue to rise, at least until populations are effectively vaccinated.$^{31}$ Further research is needed to understand the mechanisms that lead to increased susceptibility and mortality in patients with lung cancer infected with SARS-CoV-2. To date, as supported by the main international worldwide oncology societies, including American Association for Cancer Research (AACR), ${ }^{73}$ ASCO, ${ }^{74}$ ESMO, ${ }^{75}{ }^{76}$ National Comprehensive Cancer Network (NCCN) and Society for Immunotherapy of Cancer (SITC) ${ }^{77}$ patients with cancer and in particular lung cancer should receive an approved COVID-19 vaccination, also for those undergoing treatments, including chemotherapy, target agents and immunotherapy. Furthermore, as the development of new therapies to treat or prevent COVID-19 infection increases, evaluating the efficacy and side effects of these therapies, and their interaction with anticancer agents including effects of the vaccines will be essential for continuing to care for patients with lung cancer.

Funding The authors have not declared a specific grant for this research from any funding agency in the public, commercial or not-for-profit sectors.

Competing interests AP received honoraria for consulting, advisory role or lectures from AstraZeneca, Agilent/Dako, Boehringer Ingelheim, Bristol-Myers Squibb, Eli Lilly, Jansenn, Merck Sharp \& Dohme, Pfizer and Roche Genentech. $\mathrm{CB}$ received honoraria for consulting from AstraZeneca, Biostrategies Group, Curio Science, Genentech, Pfizer, Seattle Genetics, Takeda; and honorarium from American Physicians Institute, Creative Educational Concepts, OncLive. MCG has received grants and personal fees from Eli Lilly, Otsuka Pharmaceutical, AstraZeneca, Novartis, BMS, Roche, Pfizer, Celgene, Incyte, Bayer, MSD, GlaxoSmithKline, Spectrum Pharmaceuticals, and Blueprint Medicines; personal fees from Boehringer Ingelheim, Inivata, Takeda, Sanofi, Seattle Genetics, DaiichiSankyo, and Janssen; grants from Tiziana Life Sciences, Clovis, Merck Serono, United Therapeutics, Merck, Turning Point Therapeutics, Ipsen, and Exelisis; and non-financial support from MSD, Pfizer, and Eli Lilly. EG reports grants from Drace, EMD Serono, Novartis, GlaxoSmithKline, Merck, Boehringer Ingelheim, Shionogi, Eisai, Bristol-Myers Squibb, ABL Bio, Sanofi, and Xilio. Research to institution: Merck, Genentech, AstraZeneca, Novartis, Lilly, Bristol-Myers Squibb, Mirati Therapeutics, Dynavax, lovance Biotherapeutics, Neon Therapeutics, and EMD serono. SP reported consultation/advisory role for AbbVie, Amgen, AstraZeneca, Bayer, Beigene, Biocartis, Boehringer Ingelheim, Bristol-Myers Squibb, Clovis, Daiichi Sankyo, Debiopharm, Eli Lilly, F. Hoffmann-La Roche, Foundation Medicine, Illumina, Incyte, Janssen, Medscape, Merck Sharp and Dohme, Merck Serono, Merrimack, Novartis, Pharma Mar, Phosphoplatin Therapeutics, Pfizer, Regeneron, Sanofi, Seattle Genetics, and Takeda; talk in a company's organized public event: AstraZeneca, Boehringer Ingelheim, Bristol-Myers Squibb, Eli Lilly, F. HoffmannLa Roche, Illumina, Medscape, Merck Sharp and Dohme, Novartis, Pfizer, Prime,
Sanofi, and Takeda; receipt of grants/research supports: (sub)investigator in trials (institutional financial support for clinical trials) sponsored by Amgen, AstraZeneca, Biodesix, Boehringer Ingelheim, Bristol-Myers Squibb, Clovis, F. Hoffmann-La Roche, GSK, Illumina, Lilly, Merck Sharp and Dohme, Merck Serono, Mirati, Novartis, and Pfizer, Phoshoplatin Therapeutics.

\section{Patient consent for publication Not required.}

Provenance and peer review Commissioned; externally peer reviewed.

Open access This is an open access article distributed in accordance with the Creative Commons Attribution Non Commercial (CC BY-NC 4.0) license, which permits others to distribute, remix, adapt, build upon this work non-commercially, and license their derivative works on different terms, provided the original work is properly cited, appropriate credit is given, any changes made indicated, and the use is non-commercial. See http://creativecommons.org/licenses/by-nc/4.0/.

\section{ORCID iD}

Antonio Passaro http://orcid.org/0000-0002-7575-3870

\section{REFERENCES}

1 Bakouny Z, Hawley JE, Choueiri TK, et al. COVID-19 and cancer: current challenges and perspectives. Cancer Cell 2020;38:629-46.

2 Zhang L, Zhu F, Xie L, et al. Clinical characteristics of COVID-19infected cancer patients: a retrospective case study in three hospitals within Wuhan, China. Ann Oncol 2020;31:894-901.

3 Saini KS, Tagliamento M, Lambertini M, et al. Mortality in patients with cancer and coronavirus disease 2019: a systematic review and pooled analysis of 52 studies. Eur J Cancer 2020;139:43-50.

4 Palmieri C, Turtle L, Docherty A, et al. 16700 prospective data of first 1,797 hospitalised patients with cancer and COVID-19 derived from the COVID-19 clinical information network and international severe acute respiratory and emerging infections Consortium, who coronavirus clinical characterisation Consortium. Ann Oncol 2020;31:S992-S973.

5 Kuderer NM, Choueiri TK, Shah DP, et al. Clinical impact of COVID-19 on patients with cancer (CCC19): a cohort study. Lancet 2020;395:1907-18

6 Horn L, Garassino M. COVID-19 in patients with cancer: managing a pandemic within a pandemic. Nat Rev Clin Oncol 2021;18:1-2.

7 Passaro A, Peters S, Mok TSK, et al. Testing for COVID-19 in lung cancer patients. Ann Oncol 2020;31:832-4.

8 Liang W, Guan W, Chen R, et al. Cancer patients in SARSCoV-2 infection: a nationwide analysis in China. Lancet Oncol 2020;21:335-7

9 Yu J, Ouyang W, Chua MLK, et al. SARS-CoV-2 transmission in patients with cancer at a tertiary care hospital in Wuhan, China. JAMA Oncol 2020;6:1108.

10 Milette S, Fiset PO, Walsh LA, et al. The innate immune architecture of lung tumors and its implication in disease progression. $J$ Pathol 2019;247:589-605.

11 Ackermann M, Verleden SE, Kuehnel M, et al. Pulmonary vascular Endothelialitis, thrombosis, and angiogenesis in Covid-19. N Engl $J$ Med 2020;383:120-8.

12 van Zyl-Smit RN, Richards G, Leone FT. Tobacco smoking and COVID-19 infection. Lancet Respir Med 2020;8:664-5.

13 Cai H. Sex difference and smoking predisposition in patients with COVID-19. Lancet Respir Med 2020;8:e20.

14 Vardavas Cl, Nikitara K. COVID-19 and smoking: a systematic review of the evidence. Tob Induc Dis 2020;18:20.

15 LYW L, Cazier JB, Starkey T. COVID-19 prevalence and mortality in patients with cancer and the effect of primary tumour subtype and patient demographics: a prospective cohort study. Lancet Oncol 2020;2020.

16 Maringe C, Spicer J, Morris M, et al. The impact of the COVID-19 pandemic on cancer deaths due to delays in diagnosis in England UK: a national, population-based, modelling study. Lancet Oncol 2020;21:1023-34.

17 Luo J, Rizvi H, Egger JV, et al. Impact of PD-1 blockade on severity of COVID-19 in patients with lung cancers. Cancer Discov 2020;10:1121-8.

18 Curigliano G. Cancer patients and risk of mortality for COVID-19. Cancer Cell 2020;38:161-3.

19 Tian J, Yuan X, Xiao J, et al. Clinical characteristics and risk factors associated with COVID-19 disease severity in patients with cancer in Wuhan, China: a multicentre, retrospective, cohort study. Lancet Oncol 2020;21:893-903. 
20 Dai M, Liu D, Liu M, et al. Patients with cancer appear more vulnerable to SARS-CoV-2: a multicenter study during the COVID-19 outbreak. Cancer Discov 2020;10:783-91.

21 Rugge M, Zorzi M, Guzzinati S. SARS-CoV-2 infection in the Italian Veneto region: adverse outcomes in patients with cancer. Nat Cancer 2020;1:784-8.

22 de Joode K, Dumoulin DW, Tol J, et al. Dutch oncology COVID-19 Consortium: outcome of COVID-19 in patients with cancer in a nationwide cohort study. Eur J Cancer 2020;141:e184

23 Lee LYW, Cazier J-B, Starkey T, et al. COVID-19 prevalence and mortality in patients with cancer and the effect of primary tumour subtype and patient demographics: a prospective cohort study. Lancet Oncol 2020;21:1309-16.

24 Rini $\mathrm{BI}$, on behalf of the CCC-19 Consortium. Understanding the impact of COVID in cancer patients through the COVID-19 and Cancer (CCC-19) and other COVID consortiums [abstract]. In: Proceedings of the AACR Virtual Meeting: COVID-19 and Cancer; 2020 Jul 20-22. Philadelphia (PA): AACR. Clin Cancer Res 2020;26:Abstract nr IA26.

25 Warner JL, Rubinstein S, Grivas P, et al. Clinical impact of COVID-19 on patients with cancer: data from the COVID-19 and cancer Consortium (CCC19). J Clin Oncol 2020;38:LBA110.

26 Grivas P, Warner J, Shyr Y, et al. LBA72 assessment of clinical and laboratory prognostic factors in patients with cancer and SARSCoV-2 infection: the COVID-19 and cancer Consortium (CCC19). Ann Oncol 2020;31:S1202-3.

27 Luo J, Rizvi H, Preeshagul IR, et al. COVID-19 in patients with lung cancer. Ann Oncol 2020;31:S0923-7534(20)39894-X.

28 Garassino MC, Whisenant JG, Huang L-C, et al. COVID-19 in patients with thoracic malignancies (TERAVOLT): first results of an international, registry-based, cohort study. Lancet Oncol 2020;21:914-22.

29 Baena Espinar J, Torri V, Whisenant J, et al. LBA75 defining COVID-19 outcomes in thoracic cancer patients: TERAVOLT (thoracic cancERs international coVid 19 collaboration). Annals of Oncology 2020;31:S1204-5.

30 Horn L, Whisenant JG, Torri V. Thoracic cancers international COVID-19 collaboration (TERAVOLT): impact of type of cancer therapy and COVID therapy on survival. Journal of Clinical Oncology;38.

31 Calles A, Aparicio MI, Alva M, et al. Outcomes of COVID-19 in patients with lung cancer treated in a tertiary hospital in Madrid. Front Oncol 2020;10:1777.

32 Dingemans A-MC, Soo RA, Jazieh AR, et al. Treatment guidance for patients with lung cancer during the coronavirus 2019 pandemic. $J$ Thorac Oncol 2020;15:1119-36.

33 Singh AP, Berman AT, Marmarelis ME, et al. Management of lung cancer during the COVID-19 pandemic. JCO Oncol Pract 2020:16:579-86.

34 Curigliano G, Banerjee S, Cervantes A, et al. Managing cancer patients during the COVID-19 pandemic: an ESMO multidisciplinary expert consensus. Ann Oncol 2020;31:1320-35.

35 Passaro A, Addeo A, Von Garnier C, et al. ESMO management and treatment adapted recommendations in the COVID-19 era: lung cancer. ESMO Open 2020;5:e000820.

36 Lei S, Jiang F, Su W, et al. Clinical characteristics and outcomes of patients undergoing surgeries during the incubation period of COVID-19 infection. EClinicalMedicine 2020;21:100331.

37 COVIDSurg Collaborative. Mortality and pulmonary complications in patients undergoing surgery with perioperative SARS-CoV-2 infection: an international cohort study. Lancet 2020;396:27-38.

38 Gourd E. Lung cancer control in the UK hit badly by COVID-19 pandemic. Lancet Oncol 2020;21:1559.

39 United Kingdom Lung Cancer Coalition. COVID-19 Matters. Available: https://www.uklcc.org.uk/wp-content/uploads/2020/10/ UKLCC-COVID-19-Matters-Report-Oct-2020.pdf [Accessed 10 Dec 2020].

40 American Society of Oncology. COVID-19 patient care information. Available: https://www.asco.org/asco-coronavirusi nformation/care-individuals-cancer-during-covid-19 [Accessed 10 Dec 2020].

41 American College of Surgeons. 2020 COVID-19 guidelines for triage of thoracic patients. Available: https://www.facs.org/covid-19/ clinical-guidance/elective-case/thoracic-cancer [Accessed 10 Dec 2020].

42 Guckenberger M, Belka C, Bezjak A, et al. Practice recommendations for lung cancer radiotherapy during the COVID-19 pandemic: an ESTRO-ASTRO consensus statement. Radiother Oncol 2020;146:223-9.

43 Kumar S, Chmura S, Robinson C, et al. Alternative multidisciplinary management options for locally advanced NSCLC during the coronavirus disease 2019 global pandemic. J Thorac Oncol 2020;15:1137-46.

44 Liao Z, Rivin Del Campo E, Salem A, et al. Optimizing lung cancer radiation treatment worldwide in COVID-19 outbreak. Lung Cancer 2020;146:230-5.

45 Wise-Draper TM. Systemic cancer treatment-related outcomes in patients with SARS-CoV-2 infection: a CCC19 registry analysis. ESMO 2020.

46 Center for Disease Control. Duration of Isolation \& Precautions for Adults. Available: https://www.cdc.gov/coronavirus/2019-ncov/hcp/ duration-isolation.html [Accessed 10 Dec 2020].

47 Hanna TP, King WD, Thibodeau S, et al. Mortality due to cancer treatment delay: systematic review and meta-analysis. BMJ 2020;4:m4087.

48 Bersanelli M. Controversies about COVID-19 and anticancer treatment with immune checkpoint inhibitors. Immunotherapy 2020;12:269-73.

49 Goldstein DA, Ratain MJ, Saltz LB. Weight-based dosing of pembrolizumab every 6 weeks in the time of COVID-19. JAMA Oncol 2020. doi:10.1001/jamaoncol.2020.2493. [Epub ahead of print: 27 May 2020].

50 Zhao X, Shen J, Ivaturi V, et al. Model-based evaluation of the efficacy and safety of nivolumab once every 4 weeks across multiple tumor types. Ann Oncol 2020;31:302-9.

51 Morrissey KM, Marchand M, Patel H, et al. Alternative dosing regimens for atezolizumab: an example of model-informed drug development in the postmarketing setting. Cancer Chemother Pharmacol 2019;84:1257-67.

52 Paz-Ares L, Dvorkin M, Chen Y, et al. Durvalumab plus platinumetoposide versus platinum-etoposide in first-line treatment of extensive-stage small-cell lung cancer (CASPIAN): a randomised, controlled, open-label, phase 3 trial. Lancet 2019;394:1929-39.

53 Calabrò L, Peters S, Soria J-C, et al. Challenges in lung cancer therapy during the COVID-19 pandemic. Lancet Respir Med 2020;8:542-4.

54 Bailey C, Black JRM, Swanton C. Cancer research: the lessons to learn from COVID-19. Cancer Discov 2020;10:1263-6.

55 Medidata. The impact of COVID-19 on clinical trial sites. Available: https://www.medidata.com/wp-content/uploads/2020/09/COVID19Response9.0_Clinical-Trials_2020921_v2.pdf

56 Bakhribah H, Zeitouni M, Daghistani RA, et al. Implications of COVID-19 pandemic on lung cancer management: a multidisciplinary perspective. Crit Rev Oncol Hematol 2020;156:103120.

57 Colbert LE, Kouzy R, Abi Jaoude J, et al. Cancer research after COVID-19: where do we go from here? Cancer Cell 2020;37:637-8.

58 Hofman P. Challenges and issues surrounding the use for translational research of human samples obtained during the COVID-19 pandemic from lung cancer patients. Transl Lung Cancer Res 2020;9:1543-53.

59 Maringe C, Spicer J, Morris M, et al. The impact of the COVID-19 pandemic on cancer deaths due to delays in diagnosis in England, UK: a national, population-based, modelling study. Lancet Oncol 2020;21:1023-34.

60 Sha Z, Chang K, Mi J, et al. The impact of the COVID-19 pandemic on lung cancer patients. Ann Palliat Med 2020;9:3373-8.

61 Singer DS. NCl's work to advance cancer research while responding to the COVID-19 pandemic. Cancer Cell 2020;37:746-8.

62 Upadhaya S, Yu JX, Oliva C, et al. Impact of COVID-19 on oncology clinical trials. Nat Rev Drug Discov 2020;19:376-7.

63 Bakhribah H, Zeitouni M, Daghistani RA, et al. Implications of COVID-19 pandemic on lung cancer management: a multidisciplinary perspective. Crit Rev Oncol Hematol 2020;156:103120.

64 Webster P. How is biomedical research funding faring during the COVID-19 lockdown? Nat Med 2020.

65 Hartman HE, Sun Y, Devasia TP, et al. Integrated survival estimates for cancer treatment delay among adults with cancer during the COVID-19 pandemic. JAMA Oncol 2020;6:1881.

66 Kourie HR, Eid R, Haddad F, et al. The future of cancer research after COVID-19 pandemic: recession? Future Oncol 2020;16:1493-5.

67 Cancer Labs pivot to battle COVID-19. Cancer Discov 2020;10:634

68 Marcum M, Kurtzweil N, Vollmer C, et al. COVID-19 pandemic and impact on cancer clinical trials: an academic medical center perspective. Cancer Med 2020;9:6141-6.

69 Tolaney SM, Lydon CA, Li T, et al. The impact of COVID-19 on clinical trial execution at the Dana-Farber cancer Institute. J Natl Cancer Inst 2020;382. doi:10.1093/jnci/djaa144. [Epub ahead of print: 22 Sep 2020].

70 Waterhouse DM, Harvey RD, Hurley P, et al. Early impact of COVID-19 on the conduct of oncology clinical trials and long-term opportunities for transformation: findings from an American Society of clinical oncology survey. JCO Oncol Pract 2020;16:417-21. 
71 FDA guidance on conduct of clinical trials of medical products during the COVID-19 public health emergency. 9/2020, 2020.

Available: https://www.fda.gov/regulatory-information/search-fdaguidance-documents/fda-guidance-conduct-clinical-trials-medicalproducts-during-covid-19-public-health-emergency [Accessed 1 Dec 2020].

72 Guidance on the management of clinical trials during the COVID-19 (coronavirus) pandemic, 2020. Available: https://ec.europa.eu/health/ sites/health/files/files/eudralex/vol-10/guidanceclinicaltrials_covid19 en.pdf [Accessed 3 Dec 2020].

73 Ribas A, Sengupta R, Locke T, et al. Priority COVID-19 vaccination for patients with cancer while vaccine supply is limited. Cancer Discov 2020. doi:10.1158/2159-8290.CD-20-1817. [Epub ahead of print: 19 Dec 2020].
74 ASCO COVID-19 Vaccine \& Patients with Cancer. Available: https:// www.asco.org/asco-coronavirus-resources/covid-19-patient-careinformation/covid-19-vaccine-patients-cancer [Accessed 24 Jan 2021].

75 ESMO statements for vaccination against COVID-19 in patients with cancer. Available: https://www.esmo.org/covid-19-and-cancer/covid19-vaccination [Accessed 24 Jan 2021].

76 NCCN new guidance principles for vaccinating people with cancer against COVID-19. Available: https://www.nccn.org/about/news/ newsinfo.aspx? NewsID=2552 [Accessed 24 Jan 2021].

77 SITC statement on SARS-CoV-2 vaccination and cancer immunotherapy. Available: https://www.sitcancer.org/aboutsitc/ press-releases/2020/sitc-statement-sars-cov-2-vaccination-cancerimmunotherapy [Accessed 24 Jan 2021]. 